\title{
A variant TMPRSS2 isoform and ERG fusion product in prostate cancer with implications for molecular diagnosis
}

Jacques Lapointe ${ }^{1,2, *, \dagger}$, Young H Kim ${ }^{1, *}$, Melinda A Miller ${ }^{3}$, Chunde $\mathrm{Li}^{4,5}$, Gulsah Kaygusuz ${ }^{1, \ddagger}$, Matt van de Rijn ${ }^{1}$, David G Huntsman ${ }^{3}$, James D Brooks ${ }^{2}$ and Jonathan R Pollack ${ }^{1}$

\author{
${ }^{1}$ Department of Pathology, Stanford University, Stanford, CA, USA; ${ }^{2}$ Department of Urology, Stanford \\ University, Stanford, CA, USA; ${ }^{3}$ Department of Pathology, British Columbia Cancer Agency, Vancouver, BC, \\ Canada; ${ }^{4}$ Department of Oncology-Pathology, Karolinska Institutet, Stockholm, Sweden and ${ }^{5}$ Department of \\ Oncology, Karolinska University Hospital, Stockholm, Sweden
}

\begin{abstract}
Prostate cancer is the most commonly diagnosed cancer among men in the United States. Recently, fusion of TMPRSS2 with ETS family oncogenic transcription factors has been identified as a common molecular alteration in prostate cancer, where most often the rearrangement places ERG under the androgen-regulated transcriptional control of TMPRSS2. Here, we carried out rapid amplification of cDNA ends (RACE) on a prostate cancer specimen carrying an atypical aberration discovered by array-based comparative genomic hybridization (array CGH), suggesting an alternative fusion partner of ERG. We identified novel transcribed sequences fused to ERG, mapping $4 \mathrm{~kb}$ upstream of the TMPRSS2 start site. The sequences derive from an apparent second TMPRSS2 isoform, which we found also expressed in some prostate tumors, suggesting similar androgenregulated control. In a reverse transcription-polymerase chain reaction (RT-PCR)-based survey of 63 prostate tumor specimens (54 primary and nine lymph node metastases), 44 (70\%) cases expressed either the known or novel variant TMPRSS2-ERG fusion, 28 (44\%) expressed both, $10(16 \%)$ expressed only the known, and notably six $(10 \%)$ expressed only the variant isoform fusion. In this specimen set, the presence of a TMPRSS2-ERG fusion showed no statistical association with tumor stage, Gleason grade or recurrence-free survival. Nonetheless, the discovery of a novel variant TMPRSS2 isoform-ERG fusion adds to the characterization of ETS-family rearrangements in prostate cancer, and has important implications for the accurate molecular diagnosis of TMPRSS2-ETS fusions.

Modern Pathology (2007) 20, 467-473. doi:10.1038/modpathol.3800759; published online 2 March 2007
\end{abstract}

Keywords: prostate cancer; TMPRSS2-ERG fusion; molecular diagnosis

Prostate cancer is the most frequently diagnosed cancer among men in the United States, ${ }^{1}$ with one in six men being diagnosed in their lifetime. Localized prostate cancer can be treated by surgical resection or radiation, but recurs in approximately a quarter of patients. $^{2}$ Advanced prostate cancer is treated by androgen-ablation therapy, though hormone-refractory

Correspondence: Dr JR Pollack, MD, PhD, Department of Pathology, Stanford University School of Medicine, 269 Campus Drive, CCSR-3245A, Stanford, CA 94305-5176, USA.

E-mail: pollack1@stanford.edu

*These authors contributed equally to this work.

Current address: Urology Division, Department of Surgery, McGill University, Montreal, QC, Canada.

${ }^{\ddagger}$ Current address: Department of Pathology, Ankara University School of Medicine, Ankara, Turkey.

Received 29 November 2006; revised 16 January 2007; accepted 17 January 2007; published online 2 March 2007 prostate cancer invariably recurs within 1-2 years. ${ }^{3}$ Although taxane-based therapies have recently shown promise in prolonging survival, ${ }^{4}$ new therapeutic targets and molecularly directed therapies for endstage prostate cancer are urgently needed.

Recently, by examining outlier values of gene expression in human cancers, Tomlins et $a l^{5}$ discovered elevated expression of the ETS family members ERG (v-ets erythroblastosis virus E26 oncogene like) and ETV1 (Ets variant gene 1) to be a common feature of prostate cancer. Elevated expression resulted from chromosomal rearrangement fusing TMPRSS2 (transmembrane protease, serine 2) (chr 21q22.3) to $E R G$ (chr 21q22.2), an apparent intra-chromosomal deletion of $\sim 3 \mathrm{Mb}$ on chromosome 21, or less frequently to ETV1, an interchromosomal rearrangement between chr 21q22.3 and chr 7p21.2. 
ETS transcription factors are both positive and negative regulators of gene expression, and are involved in various biological processes including cell proliferation, differentiation, development, transformation and apoptosis. ${ }^{6}$ In human cancers, ETS genes are frequently found amplified, overexpressed or rearranged, the latter including chimeric fusion products characteristic of Ewing's sarcoma and certain leukemias. ${ }^{6}$ TMPRSS2, which encodes a serine protease with a transmembrane domain, ${ }^{7}$ is highly expressed in both normal and neoplastic prostate epithelium, ${ }^{8,9}$ and its expression in prostate cancer cells is androgen-regulated. ${ }^{8,10} \mathrm{In}$ prostate tumors harboring rearrangements, most frequently the non-coding first exon of TMPRSS2 has been found fused to the fourth exon of ERG in the expressed transcript. The rearrangement thereby places $E R G$ under the androgen-regulated transcriptional control of TMPRSS2. ${ }^{5}$ This finding has immediately suggested an additional possible mechanism for the androgen-dependent growth of therapy-naïve prostate tumors.

Here, we describe the discovery in prostate tumors of novel sequences fused to $E R G$, which we define as a variant TMPRSS2 isoform-ERG fusion. Our findings further our knowledge of ETS family rearrangements in prostate cancer, and provide for the more accurate molecular diagnosis of this lesion for future molecularly directed therapies.

\section{Materials and methods}

\section{Prostate Tumor Specimens}

The prostate tumor specimens included in this study, along with detailed clinicopathological annotations and procedures for specimen processing, were described previously. ${ }^{11}$ Briefly, 54 freshly frozen primary prostate tumor specimens (obtained from radical prostatectomy) and nine therapy-naive metastatic pelvic lymph node specimens (from aborted surgery) were scalpel-dissected such that $\geq 50 \%$ of cells were epithelial, and $\geq 90 \%$ of epithelial cells were neoplastic. Total RNA was extracted using the Trizol (Invitrogen, Carlsbad, CA, USA) method.

\section{RACE}

$5^{\prime}$ RNA ligase-mediated rapid amplification of cDNA ends (RACE) was performed using the SMART RACE cDNA Amplification kit (Clontech, Mountain View, CA, USA) according to the manufacturer's instructions, using a gene-specific primer from exon 7 of ERG (GCTGCACCCCCTGTGTTTCTAGCATG CATTAACCG). The PCR products were resolved by electrophoresis on a 1\% agarose gel, excised and TOPO-TA cloned into pCR4-TOPO (Invitrogen). Purified plasmid DNA from eight independent colonies was bi-directionally sequenced using M13 forward and reverse primers on an ABI 3100 automated capillary DNA sequencer by the Stanford PAN facility.

\section{RT-PCR}

RT-PCR was performed as described previously ${ }^{12}$ to characterize expression of TMPRSS2 isoforms, and the known and variant TMPRSS2-ERG fusions. Briefly, cDNA was synthesized by oligo(dT)-priming using SuperScript First-Strand Synthesis System (Invitrogen). PCR was then carried out using 1/25th the $\mathrm{RT}$ reaction product for template, $1 \times$ AmpliTaq PCR buffer, $200 \mu \mathrm{M}$ dNTPs, $1.5 \mathrm{mM} \mathrm{MgCl}_{2}, 10 \mathrm{pmol}$ of each gene-specific primer pair (Table S1, Supplementary Information), and $2 \mathrm{U}$ AmpliTaq DNA polymerase in a $50 \mu \mathrm{l}$ reaction for 35-40 cycles (see Table S1, Supplementary Information for primerspecific annealing/extension conditions), followed by electrophoresis of $1 / 10$ th the PCR product on a $1 \%$ TAE agarose gel, and imaging using an AlphaImager 2200 (Alpha Innotech, San Leandro, CA, USA).

\section{Fluorescence In Situ Hybridization}

Fluorescence in situ hybridization (FISH) was carried out on paraffin sections of selected prostate tumor cases arrayed in tissue microarray (TMA) format, as described previously. ${ }^{12}$ A $6 \mu \mathrm{M}$ TMA section was baked overnight at $60^{\circ} \mathrm{C}$, then deparaffinized in xylene and denatured in $100 \%$ ethanol. The TMA was then subjected to sequential pretreatment washes: $0.2 \mathrm{~N} \mathrm{HCl}$ for $20 \mathrm{~min}, \mathrm{ddH}_{2} \mathrm{O}$ for $10 \mathrm{~min}, 2 \times \mathrm{SSC}$ for $3 \mathrm{~min}$ and $1 \mathrm{M} \mathrm{NaSCN}$ at $80^{\circ} \mathrm{C}$ for $30 \mathrm{~min}$. Slides were then protease-treated at $37^{\circ} \mathrm{C}$ for $10 \mathrm{~min}$, dehydrated in $100 \%$ ethanol and airdried. FISH analysis was performed as described (Rajput et $a l^{13}$ ) using the following BACs (BACPAC Resources Centre, Children's Hospital Oakland Research Institute, Oakland, CA, USA): RP11-95I21 (5' $E R G), \mathrm{RP} 11-476 \mathrm{D} 17$ (3' $E R G)$ and RP11-35C4 (telomeric to TMPRSS2). BACs RP11-95I21 and RP11-35C4 were directly labeled by nick translation using Spectrum Green and Orange, respectively (Vysis, Downer's Grove, IL, USA). BAC RP11476D17 was indirectly labeled using a modified protocol with Cy5 (MetaSystems, Belmont, MA, USA) using the BioPrime DNA labeling system (Invitrogen). FISH signals were visualized on a Zeiss Axioplan epifluorescent microscope, and captured using the ISIS FISH imaging software (MetaSystems, Belmont, MA, USA); signals were counted for 50 tumor cells per specimen.

\section{Results}

In an array-based comparative genomic hybridization (array CGH) study of prostate tumors (paper in 
a TMPRSS2 (isoform 2) $\rightarrow$

TTCGCTTGCTGTTTCTGTGACTTTACGCTCTGCTGACCTAGAGGTCTTAGTTCCGGAGGG 60

AGGAATGCTGCCACCAGGAGACACAACAATGATTCAATTAAACTAGAATTTACGACTGCC 120 ACCTGGCCACGCTGAGCTCCACATGCCTCTGAATCAAAAGGCAAAGAGAGAGTTATGCAT 180 TGGCTGGGGAGACCCATCTGGACTACCAAGGAGAAGCTATAGACTACTTCTACTCCACCA 240 $E R G \longrightarrow$

GGAA GAAGCCTTATCAGTTGTGAGTGAGGACCAGTCGTTGTTTGAGTGTGCCTACGGAA 300 CGCCACACCTGGCTAAGACAGAGATGACCGCGTCCTCCTCCAGCGACTATGGACAGACTT 360 CCAAGATGAGCCCACGCGTCCCTCAGCAGGATTGGCTGTCTCAACCCCCAGCCAGGGTCA 420 CCATCAAAATGGAATGTAACCCTAGCCAGGTGAATGGCTCAAGGAACTCTCCTGATGAAT 480 GCAGTGTGGCCAAAGGCGGGAAGATGGTGGGCAGCCCAGACACCGTTGGGATGAACTACG 540 GCAGCTACATGGAGGAGAAGCACATGCCACCCCCAAACATGACCACGAACGAGCGCAGAG 600 TTATCGTGCCAGCAGATCCTACGCTATGGAGTACAGACCATGTGCGGCAGTGGCTGGAGT 660 GGGCGGTGAAA

b

b

ESTs corresponding to additional putative TMPRSS2 isoforms
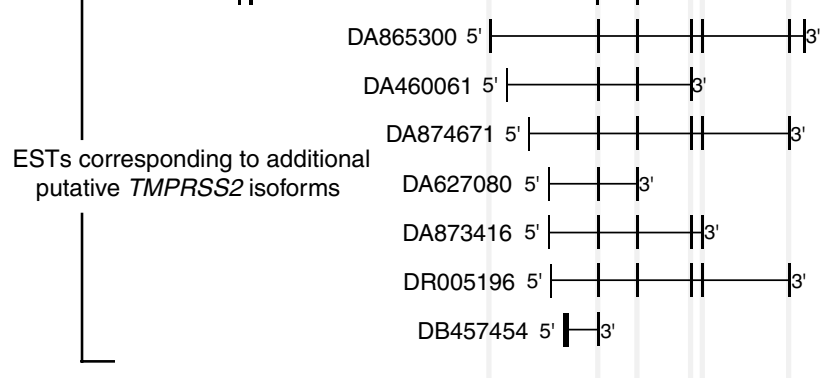

DA627080 5

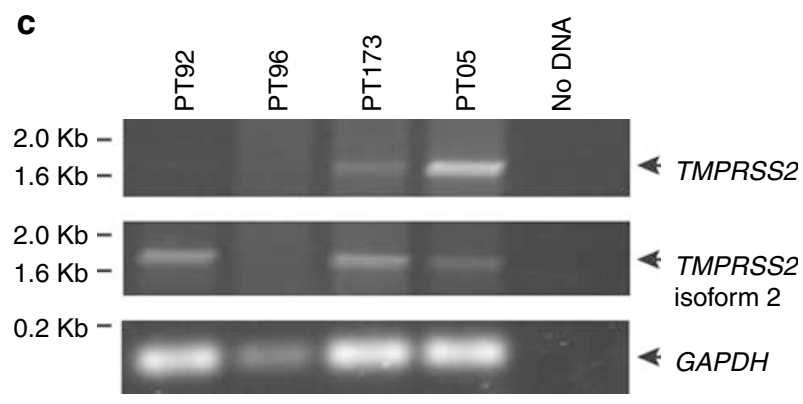


preparation), we identified a single specimen (PT187) with an apparent single-copy terminal deletion of chr 21q with the break point at $E R G$ (21q22. 2), suggesting a possible inter-chromosomal rearrangement and alternative fusion partner of $E R G$. To identify the $5^{\prime}$ fusion partner of $E R G$ in this specimen, we carried out RACE, using a $3^{\prime}$ primer within the ERG gene. The resultant PCR products, a predominant band $(\sim 0.9 \mathrm{~kb})$ and a lesser band ( $\sim 0.8 \mathrm{~kb}$ ), were separately gel-purified, cloned and sequenced. The lower molecular-weight (MW) band corresponded to the previously reported TMPRSS2 (exon1)-ERG (exon4) fusion, ${ }^{5}$ whereas four independent clones from the higher-MW band represented an identical novel sequence (Figure 1a) fused to the fourth exon of ERG. A BLAST search of this sequence to the human expressed sequence tag (EST) database yielded a match with the first exon of three overlapping ESTs (DA872508, DA868984 and DA870830; cloned from prostate tissue), mapping $4 \mathrm{~kb}$ upstream (ie distal on chr 21) of exon 1 of TMPRSS2 (Figure 1b).

The genome annotation (http://genome.ucsc.edu) suggested these sequences to encompass an alternative start site of a variant of TMPRSS2 that included an alternate noncoding exon 1, along with known exons 2 and 3 of TMPRSS2. To define further the novel transcript, we carried out RT-PCR analysis using a $5^{\prime}$ primer within the novel sequences (Figure $1 \mathrm{a})$, and a $3^{\prime}$ primer from the terminal exon (exon 14) of TMPRSS2. We identified an expressed transcript in three of four prostate tumors analyzed (Figure 1c), with a size consistent with its comprising an alternative exon 1 but sharing the known exons 214 of TMPRSS2 (Figure 1b). We therefore propose to name the novel non-rearranged transcript variant, TMPRSS2 isoform 2.

In the index prostate tumor specimen on which we carried out RACE, we identified noncoding exon 1 of the variant TMPRSS2 isoform 2 fused to the fourth exon of ERG. To confirm the expression and assess the frequency of the variant TMPRSS2 iso- form- $E R G$ fusion in prostate cancer, we used RT-PCR primer sets (Figure 1b; Table S1, Supplementary Information) designed to amplify specifically either the known or variant fusion transcripts. Amplification of GAPDH served as a positive control. Altogether, we surveyed 63 prostate tumors, including 54 primary prostate tumors and nine unmatched (ie from different patients) therapy-naïve lymph node metastases, for which we had previously profiled gene expression by cDNA microarray. ${ }^{11}$ Exemplary data are shown in Figure 2. Of the 63 specimens, $44(70 \%)$ cases expressed either the known or novel variant TMPRSS2-ERG fusion, and $28(44 \%)$ expressed both. Ten cases (16\%) expressed only the known fusion, and notably six cases $(10 \%)$ expressed only the novel variant isoform fusion (eg Figure 2, specimens PT112 and PL114).

The co-expression of both the known and variant fusions in a subset of specimens might reflect alternative transcriptional start sites from the same rearranged chromosome, or alternatively disparate start sites from two different rearranged chromosomes. To distinguish between these possibilities, we carried out FISH analysis on paraffin sections corresponding to four prostate tumor specimens co-expressing the known and variant fusions. In two of the four specimens analyzed, only a single (rather than multiple) TMPRSS2-ERG chromosomal rearrangement was detected in the majority of cells (Figure 3), indicating that both transcripts could be expressed from the same rearranged locus.

Scoring the known and variant TMPRSS2-ERG fusions together, TMPRSS2-ERG expression showed no statistical association with specimen type (primary vs metastasis), tumor stage or Gleason grade (Table 1). We also found no association with recurrence-free survival time (Figure S1, Supplementay Information), though the number of specimens with clinical follow-up $(n=28)$, and the median clinical follow up time ( 2 years) were both modest.

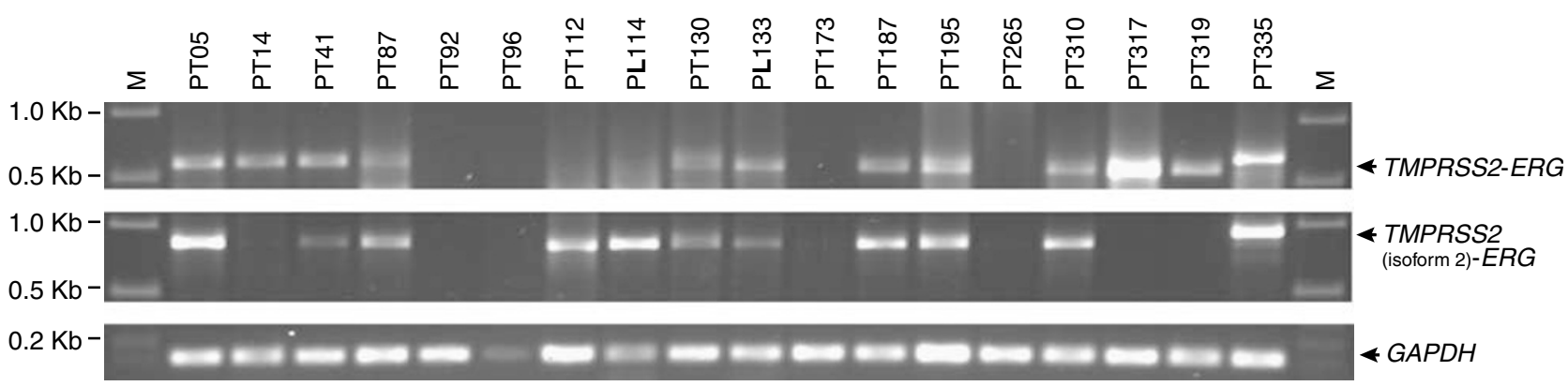

Figure 2 Expression of the variant TMPRSS2 (isoform 2)-ERG fusion in prostate cancer. Shown are representative RT-PCR assays scoring the expression of known (top panel) and variant isoform (middle panel) TMPRSS2-ERG fusion transcripts in prostate cancer, along with GAPDH control (lower panel). Molecular sizes (kb) are indicated. PT and PL indicate primary tumor and lymph node metastasis samples, respectively. PCR product sizes are consistent with, and limited DNA sequence analysis confirms that most fusions represent TMPRSS2 (exon1)-ERG (exon4) transcripts (top panel) and TMPRSS2 (isoform 2, exon1)-ERG (exon4) transcripts (middle panel). The higher-MW PCR product from sample PT335 corresponds to fusion of TMPRSS2 (exon1) to ERG (exon3), and a lower-MW PCR product from sample PT316 (not shown) corresponds to fusion of TMPRSS2 (exon2) to ERG (exon5); both variants have been previously reported. ${ }^{15,17}$ 

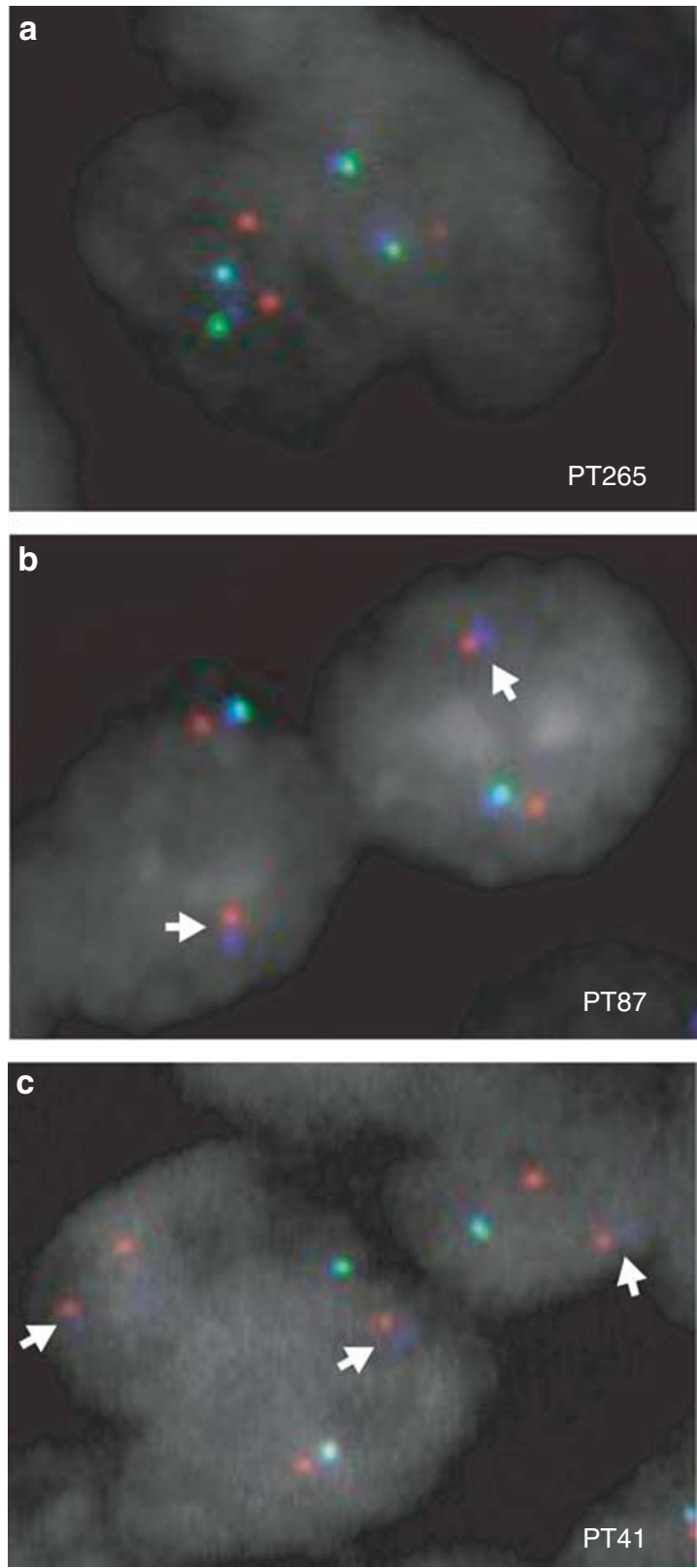

Figure 3 FISH analysis of TMPRSS2-ERG rearrangement. Representative FISH images from prostate tumors determined by RTPCR to express (a) no TMRPSS2-ERG fusion (PT265), and (b, c) both known and novel variant isoform TMPRSS2-ERG fusion transcripts (PT87, PT41). FISH signals in these pseudocolored images are red (just telomeric to TMPRSS2; RP11-35C4), green $\left(5^{\prime}\right.$ ERG; RP11-95I21) and blue (3'ERG; RP11-476D17). Rearrangement (arrows) is evident by juxtaposition of red and blue signals, typically with loss of green signal. Note that in non-rearranged chromosomes the proximity of green and blue signals can result in an aqua-colored signal.

\section{Discussion}

Our report represents an independent confirmation of the high frequency of TMPRSS2-ERG fusion in prostate cancer. Scored together, the known and/or variant fusion was expressed in $70 \%$ of cases assayed. These findings are comparable to the original report by Tomlins et $a l^{5}(79 \%$ of cases, scored by FISH), and recent confirmations by Soller et $a l^{14}$ (78\%, RT-PCR), Yoshimoto et $a l^{15}$ (40\%, RTPCR), Perner et $a l^{16}$ (49\%, FISH), and Wang et $a l^{17}$ ( $59 \%$, RT-PCR). However, it should be noted that our set of cases was selected in part for sufficient scalpel-dissected tumor material for expression profiling (before the availability of robust methods for RNA amplification). Therefore, it is biased towards larger specimens, and our result should not be regarded as a true population estimate.

In our set of specimens, we found no relationship between TMPRSS2-ERG expression and tumor stage, Gleason grade or tumor recurrence. Although the TMPRSS2 (exon 1)-ERG (exon 4) fusion is the most common, other expressed variants have been reported, ${ }^{15,17}$ differing with respect to the exon break point sites in TMPRSS2 (exons 1-5) and ERG (exons $2-5$ ). Depending on the exon break point, protein translation is predicted to start either at the native ATG of ERG (exon 3), the native ATG of TMPRSS2 (exon 2), or an in-frame ATG within exon 4 or 5 of $E R G$; in the latter cases the $\mathrm{N}$ terminus of ERG would be truncated. Interestingly, the expression of selected TMPRSS2-ERG break point variants has recently been linked to specific clinicopathological features of prostate tumors. ${ }^{17}$ Although we had sequenced only a subset of fusion products in our study (see Figure 2 legend), the PCR product sizes of most are consistent with their representing the common TMPRSS2 (exon1)-ERG (exon4) and the novel TMPRSS2 (isoform2, exon1)-ERG (exon4) transcripts, for both of which protein translation is predicted to initiate from an in-frame ATG in $E R G$ exon 4.

Given that TMPRSS2 isoform 2 is expressed in prostate tumors, and that a fraction of prostate tumors harbors rearrangement of the variant TMPRSS2 (isoform 2)-ERG fusion alone (where it is a presumptive pathogenic alteration), it is likely that TMPRSS2 isoform 2 and the resultant ERG fusion are under similar androgen-regulated transcriptional control as is TMPRSS2 (isoform 1) and its fusion. However, further studies are needed to elucidate the roles of the known and variant $E R G$ fusions in the pathogenesis and androgen dependency (and subsequent independency) of prostate cancer.

Interestingly, also mapped on the genome assembly (Figure 1b, top) are additional ESTs that, like the ESTs (DA872508, DA868984 and DA870830) corresponding to TMPRSS2 isoform 2, appear likely to comprise alternative first exons and transcriptional start sites of TMPRSS2 variants. The first exons of these distinct transcripts map both upstream (DB046885; $23 \mathrm{~kb}$ upstream of TMPRSS2) and downstream (DA865300, DA460061, DA874671, DA627080, DA873416, DR005196 and DB457454) of TMPRSS2 exon 1. It is possible that these putative TMPRSS2 isoforms also serve as androgen-regulated 
Table 1 Histopathological associations of TMPRSS2-ERG fusion

\begin{tabular}{lrr}
\hline Histopathological parameter & No TMPRSS2-ERG fusion & TMPRSS2-ERG fusion \\
\hline Sample type & & \\
$\quad$ Primary $(n=54)$ & $15(28 \%)$ & $59(72 \%)$ \\
LN metastasis $(n=9)$ & $4(44 \%)$ & \\
Tumor stage $e^{\mathrm{b}, \mathrm{c}}$ & & $15(62 \%)$ \\
Local $(\leq \mathrm{T} 2)(n=24)$ & $9(38 \%)$ & $20(80 \%)$ \\
Advanced $(\geq \mathrm{T} 3)(n=25)$ & $5(20 \%)$ & \\
Gleason grade & & $15(62 \%)$ \\
$<7(n=24)$ & $9(38 \%)$ & $13(76 \%)$ \\
$\quad=7(n=17)$ & $4(24 \%)$ & $11(85 \%)$ \\
$>7(n=13)$ & $2(15 \%)$ & $0.43^{\mathrm{a}}$ \\
\hline
\end{tabular}

Abbreviation: LN, lymph node.

${ }^{\mathrm{a}}$ Fisher's exact test (two-tail).

${ }^{\mathrm{b}}$ Primary tumors only.

${ }^{\mathrm{C}}$ Information not available on all specimens.

$\mathrm{d}^{2}$ test.

fusion partners for $E R G$ (or ETV1) rearrangements. Indeed, our preliminary data (not shown) identify the additional expression of a DA460061 (exon1)ERG (exon 4) fusion in the index specimen (PT187). To note, Tomlins et $a l^{18}$ recently reported infrequent cases of prostate cancer harboring novel genomic sequences $8 \mathrm{~kb}$ upstream of TMPRSS2 (Figure $1 \mathrm{~b}$, arrowhead) fused to ETV4, also an ETS family member. These sequences, which do not match any known EST, are distinct from any of those we report here, but may also result from androgenregulated expression.

In the index prostate tumor specimen for which we carried out RACE, an apparent terminal deletion identified by array CGH suggested an inter-chromosomal rearrangement. However, RACE-PCR identified only the known and variant isoform TMPRSS2$E R G$ fusion reported here. A likely explanation is that TMPRSS2-ERG fusion in this specimen results from an unbalanced inter-chromosomal rearrangement between two copies of chr 21. Indeed, a recent study, ${ }^{16}$ and our own data (unpublished), indicate that only half or less of TMPRSS2-ERG fusion events are associated with intra-chromosomal deletion between the TMPRSS2 and ERG loci on chr 21. Whether other prostate tumor specimens might contain rearrangements fusing $E R G$ to genes other than TMPRSS2, and its isoforms described here, remains to be investigated.

Our findings underscore the pathogenetic relevance of altered ERG expression in prostate cancer, as well as the potential for novel therapeutic opportunities. Importantly, $10 \%$ of specimens in our series expressed only the variant isoform TMPRSS2-ERG fusion, which would be missed using published RT-PCR primers from the known TMPRSS2 transcript. ${ }^{5,14,15,17}$ Our discovery, therefore, has important implications for the accurate detection of TMPRSS2-ERG fusion transcript, which is desirable for defining the molecular and pathological correlates of the fusion in tumor specimens, and may be useful in the future for molecular diagnosis. Specifically, accurate detection of TMPRS22-ERG fusion transcripts in prostate tissue, or in fluids such as urine, prostatic secretions, seminal fluid, ejaculate or blood, may prove useful in the diagnosis of prostate cancer, in prognostication and selection of appropriately aggressive therapies, and possibly in the future in selection of patients for new directed therapies targeting the oncogenic fusion.

\section{Acknowledgement}

We thank members of the Pollack lab for helpful discussion. This work was supported in part by grants from the NIH (CA111782; JDB). Nucleotide sequences for the TMPRSS2 (isoform 2)-ERG fusion have been deposited at GenBank with accession number EF194202.

\section{References}

1 Jemal A, Siegel R, Ward E, et al. Cancer statistics, 2006. CA Cancer J Clin 2006;56:106-130.

2 Khan MA, Partin AW. Management of patients with an increasing prostate-specific antigen after radical prostatectomy. Curr Urol Rep 2004;5:179-187.

3 Kasamon KM, Dawson NA. Update on hormonerefractory prostate cancer. Curr Opin Urol 2004;14: 185-193.

4 Petrylak DP, Tangen CM, Hussain MH, et al. Docetaxel and estramustine compared with mitoxantrone and prednisone for advanced refractory prostate cancer. N Engl J Med 2004;351:1513-1520. 
5 Tomlins SA, Rhodes DR, Perner S, et al. Recurrent fusion of TMPRSS2 and ETS transcription factor genes in prostate cancer. Science 2005;310:644-648.

6 Seth A, Watson DK. ETS transcription factors and their emerging roles in human cancer. Eur J Cancer 2005;41:2462-2478.

7 Paoloni-Giacobino A, Chen $\mathrm{H}$, Peitsch $\mathrm{MC}$, et al. Cloning of the TMPRSS2 gene, which encodes a novel serine protease with transmembrane, LDLRA, and SRCR domains and maps to 21q22.3. Genomics 1997;44:309-320.

8 Lin B, Ferguson C, White JT, et al. Prostate-localized and androgen-regulated expression of the membranebound serine protease TMPRSS2. Cancer Res 1999; 59:4180-4184.

9 Afar DE, Vivanco I, Hubert RS, et al. Catalytic cleavage of the androgen-regulated TMPRSS2 protease results in its secretion by prostate and prostate cancer epithelia. Cancer Res 2001;61:1686-1692.

10 Jacquinet E, Rao NV, Rao GV, et al. Cloning and characterization of the cDNA and gene for human epitheliasin. Eur J Biochem 2001;268:2687-2699.

11 Lapointe J, Li C, Higgins JP, et al. Gene expression profiling identifies clinically relevant subtypes of prostate cancer. Proc Natl Acad Sci USA 2004;101: 811-816.

12 Kim H, Lapointe J, Kaygusuz G, et al. The retinoic acid synthesis gene ALDH1a2 is a candidate tumor suppressor in prostate cancer. Cancer Res 2005;65: 8118-8124.
13 Rajput AB, Miller MA, De Luca A, et al. Frequency of the TMPRSS2:ERG gene fusion is increased in moderate to poorly differentiated prostate cancers. J Clin Pathol 2007, 26 January [E-pub ahead of print].

14 Soller MJ, Isaksson M, Elfving P, et al. Confirmation of the high frequency of the TMPRSS2/ERG fusion gene in prostate cancer. Genes Chromosomes Cancer 2006;45:717-719.

15 Yoshimoto M, Joshua AM, Chilton-Macneill S, et al. Three-color FISH analysis of TMPRSS2/ERG fusions in prostate cancer indicates that genomic microdeletion of chromosome 21 is associated with rearrangement. Neoplasia 2006;8:465-469.

16 Perner S, Demichelis F, Beroukhim R, et al. TMPRSS2:ERG Fusion-Associated Deletions Provide Insight into the Heterogeneity of Prostate Cancer. Cancer Res 2006;66:8337-8341.

17 Wang J, Cai Y, Ren C, et al. Expression of variant TMPRSS2/ERG fusion messenger RNAs is associated with aggressive prostate cancer. Cancer Res 2006;66: 8347-8351.

18 Tomlins SA, Mehra R, Rhodes DR, et al. TMPRSS2:ETV4 gene fusions define a third molecular subtype of prostate cancer. Cancer Res 2006;66: 3396-3400.

19 Owczarek CM, Portbury KJ, Hardy MP, et al. Detailed mapping of the ERG-ETS2 interval of human chromosome 21 and comparison with the region of conserved synteny on mouse chromosome 16. Gene 2004;324: 65-77.

Supplementary Information accompanies the paper on the Modern Pathology website (http://www.nature. com/modpathol) 